\title{
Nonlinear Volterra Integral Equation of the Second Kind and Biorthogonal Systems
}

\author{
M. I. Berenguer, D. Gámez, A. I. Garralda-Guillem, \\ and M. C. Serrano Pérez
}

E.U. Arquitectura Técnica, Departamento de Matemática Aplicada, Universidad de Granada, c/ Severo Ochoa, s/n, 18071 Granada, Spain

Correspondence should be addressed to M. I. Berenguer, maribel@ugr.es

Received 9 April 2010; Accepted 17 June 2010

Academic Editor: D. Anderson

Copyright (C) 2010 M. I. Berenguer et al. This is an open access article distributed under the Creative Commons Attribution License, which permits unrestricted use, distribution, and reproduction in any medium, provided the original work is properly cited.

We obtain an approximation of the solution of the nonlinear Volterra integral equation of the second kind, by means of a new method for its numerical resolution. The main tools used to establish it are the properties of a biorthogonal system in a Banach space and the Banach fixed point theorem.

\section{Introduction}

This paper puts forth a new method in order to numerically solve the nonlinear Volterra integral equation of the second kind

$$
x(t)=y_{0}(t)+\int_{\alpha}^{t} K(t, s, x(s)) d s, \quad t \in[\alpha, \alpha+\beta]
$$

where $y_{0}:[\alpha, \alpha+\beta] \rightarrow \mathbb{R}$ and the kernel $K:[\alpha, \alpha+\beta]^{2} \times \mathbb{R} \rightarrow \mathbb{R}$ are assumed to be known continuous functions, and the unknown function to be determined is $x:[\alpha, \alpha+\beta] \rightarrow \mathbb{R}$.

Modeling many problems of science, engineering, physics, and other disciplines leads to linear and nonlinear Volterra integral equations of the second kind. These are usually difficult to solve analytically and in many cases the solution must be approximated. Therefore, in recent years several numerical approaches have been proposed (see, e.g., [4$8]$ ). The numerical methods usually transform the integral equation into a linear or nonlinear system that can be solved by direct or iterative methods. In a recent work [9], the authors use a new technique for solving the linear Volterra integral equation. The method is based on two 
classical analytical tools: the Geometric Series theorem and Schauder bases in a Banach space. The purpose of this paper is to develop, and generalize to the nonlinear case, an effective method for approximating the solution using biorthogonal systems and another classical tool in analysis: the Banach fixed point theorem.

The work is structured in three parts: in Section 2, we will recall one well-known result and some useful definitions needed later. In Section 3, we define the approximating functions and we study the error. Finally, the numerical results given in Section 4 show the high accuracy of the method.

\section{Preliminaries}

Let $C([\alpha, \alpha+\beta])$ be the Banach space of all continuous and real-valued functions on $[\alpha, \alpha+\beta]$, endowed with its usual supnorm. Let us start by observing that (1.1) is equivalent to the problem of finding fixed points of the operator

$$
T: C([\alpha, \alpha+\beta]) \longrightarrow C([\alpha, \alpha+\beta])
$$

defined by

$$
(T x)(t):=y_{0}(t)+\int_{\alpha}^{t} K(t, s, x(s)) d s, \quad t \in[\alpha, \alpha+\beta], x \in C([\alpha, \alpha+\beta]) .
$$

To establish the existence of fixed points of (2.2), we will use the version of the Banach fixedpoint theorem (see [10]) which we enunciate below: let $(X,\|\cdot\|)$ be a Banach space, let $F: X \rightarrow X$ and let $\left\{\mu_{n}\right\}_{n \geq 1}$ be a sequence of nonnegative real numbers such that the series $\sum_{n \geq 1} \mu_{n}$ is convergent and for all $x, y \in X$ and for all $n \geq 1,\left\|F^{n} x-F^{n} y\right\| \leq \mu_{n}\|x-y\|$. Then $F$ has a unique fixed point $u \in X$. Moreover, if $\bar{x}$ is an element in $X$, then we have that for all $n \geq 1$,

$$
\left\|F^{n} \bar{x}-u\right\| \leq\left(\sum_{i=n}^{\infty} \mu_{i}\right)\|F \bar{x}-\bar{x}\| .
$$

In particular, $u=\lim _{n} F^{n}(\bar{x})$.

On the other hand, we recall briefly some definitions on the theory of Schauder bases and biorthogonal systems in general (see [11]), which are central areas of research, and also some important tools in Functional Analysis. The use of Schauder bases in the numerical study of integral and differential equations has been previously considered in [12-14].

Let us start by recalling the notion of biorthogonal system of a Banach space. Let $X$ be a Banach space and $X^{*}$ its topological dual space. A system $\left\{x_{n}, f_{n}\right\}_{n \geq 1}$, where $x_{n} \in X$, $f_{n} \in X^{*}$, and $f_{n}\left(x_{m}\right)=\delta_{n m}$ ( $\delta$ is Kronecker's delta), is called a biorthogonal system in $X$. We say that the system is a fundamental biorthogonal system if $\overline{\operatorname{span}}\left\{x_{n}\right\}_{n \geq 1}=X$.

We will work with a particular type of fundamental biorthogonal systems. Let us recall that a sequence $\left\{x_{n}\right\}_{n \geq 1}$ of elements of a Banach space $X$ is called a Schauder basis of $X$ if for every $z \in X$ there is a unique sequence $\left\{\lambda_{n}\right\}_{n \geq 1}$ of scalars such that $z=\sum_{n \geq 1} \lambda_{n} x_{n}$. A Schauder basis gives rise to the canonical sequence of (continuous and linear) finite dimensional projections $P_{n}: X \rightarrow X, P_{n}\left(\sum_{n \geq 1} \lambda_{n} x_{n}\right)=\sum_{k=1}^{n} \lambda_{k} x_{k}$ and the associated sequence 
of (continuous and linear) coordinate functionals $\left\{x_{n}^{*}\right\}_{n \geq 1}$ in $X^{*}$ is given by $x_{n}^{*}\left(\sum_{n \geq 1} \lambda_{n} x_{n}\right)=$ $\lambda_{n}$. Note that a Schauder basis is always a fundamental biorthogonal system, under the interpretation of the coordinate functionals as biorthogonal functionals.

\section{Main Results}

We begin this section making use of a Schauder basis in the Banach space $C\left([\alpha, \alpha+\beta]^{2}\right)$ endowed with its usual supnorm. To construct such a basis, we recall that a usual Schauder basis $\left\{b_{n}\right\}_{n \geq 1}$ in $C([\alpha, \alpha+\beta])$ can be obtained from a dense sequence $\left\{t_{n}\right\}_{n \geq 1}$ of distinct points from $[\alpha, \alpha+\beta]$ such that $t_{1}=\alpha$ and $t_{2}=\alpha+\beta$. We set $b_{1}(t):=1$ for $t \in[\alpha, \alpha+\beta]$, and for $n \geq 1$, we let $b_{n}$ be a piecewise linear continuous function on $[\alpha, \alpha+\beta]$ with nodes at $\left\{t_{j}: 1 \leq j \leq n\right\}$, uniquely determined by the relations $b_{n}\left(t_{n}\right)=1$ and $b_{n}\left(t_{k}\right)=0$ for $k<n$. For this basis, the sequence of biorthogonal functionals $\left\{b_{n}^{*}\right\}_{n \geq 1}$ satisfies (see [1]) for all $y \in C([\alpha, \alpha+\beta]$ )

$$
b_{1}^{*}(y)=y\left(t_{1}\right), \quad b_{n}^{*}(y)=y\left(t_{n}\right)-\sum_{k=1}^{n-1} b_{k}^{*}(y) b_{k}\left(t_{n}\right), \quad \text { for } n \geq 2
$$

In addition, the sequence of associated projections $\left\{P_{n}\right\}_{n \geq 1}$ satisfies for all $y \in C([\alpha, \alpha+\beta])$, for all $n \geq 1$ and for all $k \leq n$ such that $P_{n}(y)\left(t_{k}\right)=y\left(t_{k}\right)$.

From the Schauder basis $\left\{b_{n}\right\}_{n \geq 1}$ in $C([\alpha, \alpha+\beta])$, we can build another Schauder basis $\left\{B_{n}\right\}_{n \geq 1}$ of $C\left([\alpha, \alpha+\beta]^{2}\right)$ (see $\left.[1,2]\right)$. It is sufficient to consider $B_{n}(t, s):=b_{i}(t) b_{j}(s)$ for all $t, s \in[\alpha, \alpha+\beta]$, with $\sigma(n)=(i, j)$, where for a real number $a$, [a] will denote its integer part and $\sigma=\left(\sigma_{1}, \sigma_{2}\right): \mathbb{N} \rightarrow \mathbb{N} \times \mathbb{N}$ is the bijective mapping defined by

$$
\sigma(n):= \begin{cases}(\sqrt{n}, \sqrt{n}) & \text { if }[\sqrt{n}]=\sqrt{n}, \\ \left(n-[\sqrt{n}]^{2},[\sqrt{n}]+1\right) & \text { if } 0<n-[\sqrt{n}]^{2} \leq[\sqrt{n}], \\ \left([\sqrt{n}]+1, n-[\sqrt{n}]^{2}-[\sqrt{n}]\right) & \text { if }[\sqrt{n}]<n-[\sqrt{n}]^{2} .\end{cases}
$$

Remark 3.1. The Schauder basis $\left\{B_{n}\right\}_{n \geq 1}$ of $C\left([\alpha, \alpha+\beta]^{2}\right)$ has similar properties to the ones for the one-dimensional case.

(a) For all $t, s \in[\alpha, \alpha+\beta], B_{1}(t, s)=1$ and for $n \geq 2$,

$$
B_{n}\left(t_{i}, t_{\mathrm{j}}\right)= \begin{cases}1 & \text { if } \sigma(n)=(i, j) \\ 0 & \text { if } \sigma^{-1}(i, j)<n\end{cases}
$$

(b) If $z \in C\left([\alpha, \alpha+\beta]^{2}\right)$, then $B_{1}^{*}(z)=z\left(t_{1}, t_{1}\right)$, and for all $n \geq 2$, if $\sigma(n)=(i, j), B_{n}^{*}(z)=$ $z\left(t_{i}, t_{j}\right)-\sum_{k=1}^{n-1} B_{k}^{*}(z) B_{k}\left(t_{i}, t_{j}\right)$.

(c) The sequence of associated projections $\left\{Q_{n}\right\}_{n \geq 1}$ satisfies $Q_{n}(z)\left(t_{i}, t_{j}\right)=z\left(t_{i}, t_{j}\right)$, whenever $n, i, j \in \mathbb{N}$ and $\sigma^{-1}(i, j) \leq n$.

(d) This Schauder basis is monotone, that is, $\sup \left\{\left\|Q_{n}\right\|\right\}_{n \in \mathbb{N}}=1$. 
Remark 3.2. We have chosen the Schauder basis above for simplicity in the exposition, although the method to be presented also works considering any fundamental biorthogonal system in $C\left([\alpha, \alpha+\beta]^{2}\right)$.

With the previous notation, our first result enables us to obtain the image under operator $T$ defined in (2.2) of any continuous function in terms of certain sequences of scalars, sequences which are obtained just by evaluating some functions at adequate points.

Proposition 3.3. Let $T: C([\alpha, \alpha+\beta]) \rightarrow C([\alpha, \alpha+\beta])$ be the continuous integral operator defined in (2.2). Let $x \in C[\alpha, \alpha+\beta]$, and let us consider the function $\Phi \in C\left([\alpha, \alpha+\beta]^{2}\right)$, defined by $\Phi(t, s)=K(t, s, x(s))$. Let $\left\{\lambda_{n}\right\}_{n \geq 1}$ be the sequences of scalars satisfying $\Phi=\sum_{n \geq 1} \lambda_{n} B_{n}$. Then for all $t \in[\alpha, \alpha+\beta]$, we have that

$$
(T x)(t)=y_{0}(t)+\sum_{n \geq 1} \lambda_{n} \int_{\alpha}^{t} B_{n}(t, s) d s,
$$

where

$$
\lambda_{1}=\Phi\left(t_{1}, t_{1}\right)
$$

and for $n \geq 2$,

$$
\lambda_{n}=\Phi\left(t_{i}, t_{j}\right)-\sum_{k=1}^{n-1} B_{k}^{*}(\Phi) B_{k}\left(t_{i}, t_{j}\right) \quad \text { with } \sigma(n)=(i, j)
$$

Proof. The result follows directly from the expression

$$
\Phi(t, s)=\sum_{n \geq 1} B_{n}^{*}(\Phi) B_{n}(t, s)
$$

in the integral appearing in the definition of $T$.

On the other hand, in order to discuss the application of Banach's fixed point theorem to find a fixed point for the operator $T$ defined in (2.2), we establish the following result.

Proposition 3.4. Assume that in (1.1) the kernel K satisfies a Lipschitz condition in its third variable:

$$
|K(t, s, x)-K(t, s, y)| \leq M|x-y| \quad \forall t, s \in[\alpha, \alpha+\beta], x, y \in \mathbb{R}
$$

for some constant $M>0$. Then the integral equation (1.1) has a unique solution $x \in C([\alpha, \alpha+\beta])$. In addition, for each $\bar{x} \in C([\alpha, \alpha+\beta])$, the sequence $\left\{T^{n} \bar{x}\right\}_{n \geq 1}$ in $C([\alpha, \alpha+\beta])$ converges uniformly to the unique solution $x$ and for all $n \geq 1$,

$$
\left\|T^{n} \bar{x}-x\right\| \leq \frac{(M \beta)^{n}}{n !} e^{M \beta}\|T \bar{x}-\bar{x}\|
$$


Proof. For all $x, y \in C([\alpha, \alpha+\beta])$ and for all $n \geq 1$, we obtain by a mathematical induction (see $\left[3\right.$, Theorem 5.2.3]) that $\left|\left(T^{n} x\right)(t)-\left(T^{n} y\right)(t)\right| \leq\left(M^{n} / n !\right)\|x-y\|(t-\alpha)^{n}$ for all $t \in[\alpha, \alpha+\beta]$. In particular,

$$
\left\|T^{n} x-T^{n} y\right\| \leq \frac{(M \beta)^{n}}{n !}\|x-y\|
$$

Since $\sum_{n \geq 1}\left((M \beta)^{n} / n !\right)$ converges for any $\beta$ and $M$, by Banach's fixed point theorem, we will derive the existence and uniqueness of a solution of the integral equation (1.1). From (3.10) and (2.3), we deduce (3.9).

In view of Propositions 3.3 and 3.4, (3.4) gives the unique solution $x(t)$ of (1.1). The problem is that generally this expression cannot be calculated explicitly. The idea of the proposed method is to truncate to calculate approximately a sequence of iterations and projections that converge to the solution. More specifically, let $\bar{x}:[\alpha, \alpha+\beta] \rightarrow \mathbb{R}$ be a continuous function, and $n_{1}, n_{2}, n_{3}, \ldots, \in \mathbb{N}$. Consider the continuous functions

$$
z_{0}(t):=\bar{x}(t), \quad t \in[\alpha, \alpha+\beta]
$$

and for $r \in \mathbb{N}$, we define

$$
\begin{gathered}
L_{r-1}(t, s):=K\left(t, s, z_{r-1}(s)\right) \quad(t, s \in[\alpha, \alpha+\beta]), \\
z_{r}(t):=y_{0}(t)+\int_{\alpha}^{t} Q_{n_{r}^{2}}\left(L_{r-1}(t, s)\right) d s \quad(t \in[\alpha, \alpha+\beta]) .
\end{gathered}
$$

In order to obtain the convergence of the sequence $\left\{z_{r}\right\}_{r \geq 1}$ to the unique solution of (1.1), we need, under some weak condition, to uniformly estimate the rate of the convergence of the sequence of projections $\left\{Q_{n}\right\}_{n \geq 1}$ in the bidimensional case. To this end, we introduce the following notation that will be used in the next results: if $\left\{t_{n}\right\}_{n \geq 1}$ is the dense subset of distinct points in $[\alpha, \alpha+\beta]$, we considered to define the Schauder basis, let $T_{n}$ be the set $\left\{t_{j}, 1 \leq j \leq n\right\}$ ordered in an increasing way for $n \geq 2$. Let $\Delta T_{n}$ denote the maximum distance between two consecutive points of $T_{n}$.

Proposition 3.5. Let $K \in C^{1}\left([\alpha, \alpha+\beta]^{2} \times \mathbb{R}\right)$ such that $K, \partial K / \partial t, \partial K / \partial s, \partial K / \partial x$ satisfy a global Lipschitz condition in the third variable. Then $\left\{\partial L_{r-1} / \partial t\right\}_{r \geq 1}$ and $\left\{\partial L_{r-1} / \partial s\right\}_{r \geq 1}$ are uniformly bounded.

Proof. From (3.12), we have that for all $r \geq 1$,

$$
\frac{\partial L_{r-1}}{\partial t}(t, s)=\frac{\partial K}{\partial t}\left(t, s, z_{r-1}(s)\right), \quad \frac{\partial L_{r-1}}{\partial s}(t, s)=\frac{\partial K}{\partial s}\left(t, s, z_{r-1}(s)\right)+\frac{\partial K}{\partial x}\left(t, s, z_{r-1}(s)\right) z_{r-1}^{\prime}(s)
$$


Let $R=\max _{(t, s) \in[\alpha, \alpha+\beta]^{2}}\|K(t, s, 0)\|$, and we have for all $r \geq 1$ and $(t, s) \in[\alpha, \alpha+\beta]^{2}$,

$$
\begin{aligned}
\left\|L_{r-1}(t, s)\right\| & =\left\|K\left(t, s, z_{r-1}(s)\right)\right\| \\
& \leq\left\|K\left(t, s, z_{r-1}(s)\right)-K(t, s, 0)\right\|+\|K(t, s, 0)\| \\
& \leq M\left\|z_{r-1}(s)\right\|+R
\end{aligned}
$$

with $M$ as the Lipschitz constant of $K$.

As a consequence of the monotonicity of the Schauder basis, we have

$$
\left\|z_{r}(t)\right\| \leq\left\|y_{0}\right\|+\int_{\alpha}^{t}\left\|L_{r-1}\left(t, t_{1}\right)\right\| d t_{1}
$$

If one applies (3.15) and by repeating the previous argument,

$$
\begin{aligned}
\left\|z_{r}(t)\right\| & \leq\left\|y_{0}\right\|+\int_{\alpha}^{t}\left(M\left\|z_{r-1}\left(t_{1}\right)\right\|+R\right) d t_{1} \\
& \leq\left\|y_{0}\right\|+\int_{\alpha}^{t}\left(M\left(\left\|y_{0}\right\|+\int_{\alpha}^{t_{1}}\left\|L_{r-2}\left(t_{1}, t_{2}\right)\right\| d t_{2}\right)+R\right) d t_{1} \\
& =\left\|y_{0}\right\|\left(1+M \int_{\alpha}^{t} d t_{1}\right)+R \int_{\alpha}^{t} d t_{1}+M \int_{\alpha}^{t} \int_{\alpha}^{t_{1}}\left\|L_{r-2}\left(t_{1}, t_{2}\right)\right\| d t_{2} d t_{1} .
\end{aligned}
$$

Applying recursively this process and the Fubini theorem, we get

$$
\left\|z_{r}(t)\right\| \leq\left\|y_{0}\right\|+\left\|y_{0}\right\| \sum_{k=1}^{r-1} \frac{M^{k}(t-\alpha)^{k}}{k !}+R \sum_{k=1}^{r-1} \frac{M^{k-1}(t-\alpha)^{k}}{k !}+M^{r-1} \frac{(t-\alpha)^{r}}{r !}\left\|L_{0}\left(t_{r-1}, t_{r}\right)\right\| .
$$

Thus for all $r \geq 1$ and $(t, s) \in[\alpha, \alpha+\beta]^{2}$,

$$
\left\|z_{r}\right\| \leq\left\|y_{0}\right\|+\left(\left\|y_{0}\right\|+\frac{R}{M}\right) \sum_{k=1}^{r-1} \frac{(\beta M)^{k}}{k !}+\frac{\left\|L_{0}\right\|}{M} \frac{(\beta M)^{r}}{r !}
$$

Hence the sequence $\left\{z_{r}\right\}_{r \geq 1}$ is uniformly bounded.

Meanwhile $z_{r}^{\prime}(t)=y_{0}^{\prime}(t)+Q_{n_{r}^{2}}\left(L_{r-1}(t, t)\right)+\int_{\alpha}^{t}\left(\partial Q_{n_{r}^{2}} / \partial t\right)\left(L_{r-1}(t, s)\right) d s$, and in view of the monotonicity of the Schauder basis $\left\{B_{n}\right\}_{n \geq 1}$ and the fact that for all $z \in C^{1}\left([\alpha, \alpha+\beta]^{2}\right)$ and $n \geq 2$ we have that $\left|\left(\partial Q_{n^{2}}(z) / \partial t\right)(t, s)\right| \leq\|\partial z / \partial t\|$, the boundedness of $\left\{z_{r}^{\prime}\right\}_{r \geq 1}$ follows from that of (3.15) and of $\left\{\partial L_{r} / \partial t\right\}_{r \geq 1}$, which is done below. 
Then

$$
\left|\frac{\partial K}{\partial t}\left(t, s, z_{r-1}(s)\right)\right| \leq\left|\frac{\partial K}{\partial t}\left(t, s, z_{r-1}(s)\right)-\frac{\partial K}{\partial t}(t, s, 0)\right|+\left|\frac{\partial K}{\partial t}(t, s, 0)\right| \leq M_{1}\left|z_{r-1}(s)\right|+U
$$

with $U=\max _{(t, s) \in[\alpha, \alpha+\beta]^{2}}|(\partial K / \partial t)(t, s, 0)|$ and $M_{1}$ being the Lipschitz constant of $(\partial K /$ $\partial t)\left(t, s, z_{r-1}(s)\right)$.

Similarly,

$$
\left|\frac{\partial K}{\partial s}\left(t, s, z_{r-1}(s)\right)\right| \leq M_{2}\left|z_{r-1}(s)\right|+V
$$

with $V=\max _{(t, s) \in[\alpha, \alpha+\beta]^{2}}|(\partial K / \partial s)(t, s, 0)|$ and $M_{2}$ as the Lipschitz constant of $(\partial K /$ $\partial s)\left(t, s, z_{r-1}(s)\right)$.

Finally

$$
\left|\frac{\partial K}{\partial x}\left(t, s, z_{r-1}(s)\right) z_{r-1}^{\prime}(s)\right| \leq\left(M_{3}\left|z_{r-1}(s)\right|+W\right)\left|z_{r-1}^{\prime}(s)\right|
$$

with $W=\max _{(t, s) \in[\alpha, \alpha+\beta]^{2}}|(\partial K / \partial x)(t, s, 0)|$ and $M_{3}$ as the Lipschitz constant of $(\partial K /$ $\partial x)\left(t, s, z_{r-1}(s)\right)$.

Hence, $\left\{\partial L_{r-1} / \partial t\right\}_{r \geq 1}$ and $\left\{\partial L_{r-1} / \partial s\right\}_{r \geq 1}$ are uniformly bounded.

Theorem 3.6. With the previous notation, let $\bar{x} \in C([\alpha, \alpha+\beta]), y_{0} \in C^{1}([\alpha, \alpha+\beta])$, and $K \in$ $C^{1}\left([\alpha, \alpha+\beta]^{2} \times \mathbb{R}\right)$ with $K, \partial K / \partial t, \partial K / \partial s, \partial K / \partial x$ satisfying the Lipschitz global condition of the third variable. Then, there is $\rho>0$ such that for all $r \geq 1$ and $n_{r} \geq 2$,

$$
\left\|L_{r-1}-Q_{n_{r}}\left(L_{r-1}\right)\right\| \leq \rho \Delta T_{n_{r}}
$$

Proof. We use Proposition 3.5. and the inequality resulting from Remark 3.1.(c) and the Mean Value Theorem to get

$$
\left\|z-Q_{n^{2}}(z)\right\| \leq 4 \max \left\{\left\|\frac{\partial z}{\partial t}\right\|,\left\|\frac{\partial z}{\partial s}\right\|\right\} \Delta T_{n}
$$

for $z \in C^{1}\left([\alpha, \alpha+\beta]^{2}\right)$ and $n \geq 2$.

The main result that establishes that the sequence defined in (3.11) and (3.13) approximates the solution of (1.1) as well as giving an upper bond of the error committed is given below. 
Theorem 3.7. Let $K \in C\left([\alpha, \alpha+\beta]^{2} \times \mathbb{R}\right)$ such that $K$ satisfies a global Lipschitz condition in the third variable and let $\bar{x} \in C([\alpha, \alpha+\beta])$. Let $m \in \mathbb{N}$, and assume that certain positive numbers $\varepsilon_{1}, \ldots, \varepsilon_{m}$ satisfy

$$
\left\|T z_{r-1}-z_{r}\right\|<\varepsilon_{r}, \quad r=1, \ldots, m
$$

and let $x$ be the exact solution of the integral equation (1.1). Then

$$
\left\|x-z_{m}\right\| \leq \frac{(M \beta)^{m}}{m !} e^{M \beta}\|T \bar{x}-\bar{x}\|+\sum_{r=1}^{m} \varepsilon_{r} \frac{(M \beta)^{m-r}}{(m-r) !}
$$

where $M$ is the Lipschitz constant of $K$.

Proof. On one hand, Proposition 3.4 gives

$$
\left\|x-T^{m} \bar{x}\right\| \leq \frac{(M \beta)^{m}}{m !} e^{M \beta}\|T \bar{x}-\bar{x}\|
$$

On the other hand, in view of (3.10) for all $r=1, \ldots, m$, we have that

$$
\left\|T^{m-r+1} z_{r-1}-T^{m-r} z_{r}\right\|=\left\|T^{m-r} T z_{r-1}-T^{m-r} z_{r}\right\| \leq \frac{(M \beta)^{m-r}}{(m-r) !}\left\|T z_{r-1}-z_{r}\right\| .
$$

Hence

$$
\left\|T^{m} \bar{x}-z_{m}\right\|=\left\|T^{m} z_{0}-z_{m}\right\| \leq \sum_{r=1}^{m}\left\|T^{m-r+1} z_{r-1}-T^{m-r} z_{r}\right\| \leq \sum_{r=1}^{m} \varepsilon_{r} \frac{(M \beta)^{m-r}}{(m-r) !} .
$$

Then we use the triangular inequality

$$
\left\|x-z_{m}\right\| \leq\left\|x-T^{m} \bar{x}\right\|+\left\|T^{m} \bar{x}-z_{m}\right\|
$$

and the proof is complete in view of (3.27) and (3.29).

Remark 3.8. Under the hypothesis of Theorem 3.6, $\left\|T z_{r-1}-z_{r}\right\|$ can be estimated as follows: there is $\rho>0$ such that for all $r \geq 1$ and $n_{r} \geq 2$,

$$
\left\|T z_{r-1}-z_{r}\right\| \leq \beta\left\|L_{r-1}-Q_{n_{r}^{2}}\left(L_{r-1}\right)\right\| \leq \beta \rho \Delta T_{n_{r}}
$$

Hence, given certain $\varepsilon_{1}, \ldots, \varepsilon_{m}>0$, we can find $m$ positive integers $n_{1}, \ldots, n_{m}$ such that $\left\|T z_{r-1}-z_{r}\right\|<\varepsilon_{r}$, and by Theorem 3.7, we can state the convergence of $\left\{z_{r}\right\}_{r \geq 1}$ and an estimation of the error. 


\section{Some Examples}

The behaviour of the method introduced above will be illustrated with the following three examples.

Example 4.1. The equation

$$
\begin{gathered}
x(t)=\frac{1}{2} t(2+t)-2 t \operatorname{arctg}(t)+\ln \left(1+t^{2}\right)+\int_{0}^{t}(-x(s)+2 \operatorname{arctg}(x(s))) d s \quad(t \in[0,1]), \\
x(0)=0
\end{gathered}
$$

has the exact solution $x(t)=t$.

Example 4.2. Consider the equation

$$
\begin{gathered}
x(t)=\frac{1}{3} t \cos \left(t^{3}\right)+t^{3}-\frac{t}{3}+\int_{0}^{t} t s^{2} \sin (x(s)) d s \quad(t \in[0,1]) \\
x(0)=0
\end{gathered}
$$

whose exact solution is $x(t)=t^{3}$.

Example 4.3. Consider the equation

$$
\begin{gathered}
x(s)=\frac{1}{2}\left(3 t-\left(1+t^{2}\right) \operatorname{arctg}(t)\right)+\int_{0}^{t} s \operatorname{arctg}(x(s)) d s \quad(t \in[0,1]), \\
x(0)=0,
\end{gathered}
$$

whose exact solution is $x(t)=t$.

To construct the Schauder basis in $C\left([0,1]^{2}\right)$, we considered the particular choice $t_{1}=0$, $t_{2}=1$, and for $n \in \mathbb{N} \cup\{0\}, t_{i+1}=(2 k+1) / 2^{n+1}$ if $i=2^{n}+k+1$ where $0 \leq k<2^{n}$ are integers. To define the sequence $\left\{z_{r}\right\}_{r \geq 1}$, we take $z_{0}(t)=1$ and $n_{r}=j$ (for all $r \geq 1$ ). In Tables 1, 2, and 3, we exhibit, for $j=9,17,33,65$, and 129, the absolute errors committed in eight representative points $\left(t_{i}\right)$ of $[0,1]$ when we approximate the exact solution $x$ by the iteration $z_{2}$. The computations associated with the examples were performed using Mathematica 7.

\section{Conclusions}

In this paper, we introduce a new numerical method which approximates the solution of the nonlinear Volterra integral equation of the second kind (1.1). Unlike what happens in the classical methods, as in the collocation one, we do not need to solve high-order nonlinear systems of algebraical equations: for our method we just calculate linear combinations of scalar obtained by evaluating adequate functions. This is done due to the properties of the Schauder basis $\left\{B_{n}\right\}_{n \geq 1}$ considered in its development. 
Table 1: Absolute errors for Example 4.1.

\begin{tabular}{lccccc}
\hline$t_{i}$ & $j=9$ & $j=17$ & $j=33$ & $j=65$ & $j=129$ \\
& $\left|z_{2}\left(t_{i}\right)-x\left(t_{i}\right)\right|$ & $\left|z_{2}\left(t_{i}\right)-x\left(t_{i}\right)\right|$ & $\left|z_{2}\left(t_{i}\right)-x\left(t_{i}\right)\right|$ & $\left|z_{2}\left(t_{i}\right)-x\left(t_{i}\right)\right|$ & $\left|z_{2}\left(t_{i}\right)-x\left(t_{i}\right)\right|$ \\
\hline 0.125 & $3.93 E-5$ & $9.71 E-6$ & $2.34 E-6$ & $5.47 E-7$ & $1.17 E-7$ \\
0.250 & $1.50 E-4$ & $3.51 E-5$ & $7.65 E-6$ & $1.36 E-6$ & $6.74 E-8$ \\
0.375 & $3.54 E-4$ & $1.01 E-4$ & $3.19 E-5$ & $1.13 E-5$ & $4.52 E-6$ \\
0.5 & $8.61 E-4$ & $2.91 E-4$ & $1.11 E-4$ & $4.73 E-5$ & $2.16 E-5$ \\
0.625 & $8.77 E-4$ & $2.73 E-4$ & $9.66 E-5$ & $3.85 E-5$ & $1.68 E-5$ \\
0.750 & $1.39 E-3$ & $4.86 E-4$ & $1.92 E-4$ & $8.38 E-5$ & $3.89 E-5$ \\
0.875 & $1.35 E-3$ & $4.31 E-4$ & $1.54 E-4$ & $6.26 E-5$ & $2.77 E-5$ \\
1 & $2.76 E-3$ & $1.04 E-3$ & $4.40 E-4$ & $2.00 E-4$ & $9.51 E-5$ \\
\hline
\end{tabular}

Table 2: Absolute errors for Example 4.2.

\begin{tabular}{lccccc}
\hline$t_{i}$ & $j=9$ & $j=17$ & $j=33$ & $j=65$ & $j=129$ \\
& $\left|z_{2}\left(t_{i}\right)-x\left(t_{i}\right)\right|$ & $\left|z_{2}\left(t_{i}\right)-x\left(t_{i}\right)\right|$ & $\left|z_{2}\left(t_{i}\right)-x\left(t_{i}\right)\right|$ & $\left|z_{2}\left(t_{i}\right)-x\left(t_{i}\right)\right|$ & $\left|z_{2}\left(t_{i}\right)-x\left(t_{i}\right)\right|$ \\
\hline 0.125 & $1.59 E-7$ & $4.71 E-8$ & $1.22 E-8$ & $3.09 E-9$ & $7.76 E-10$ \\
0.250 & $6.01 E-6$ & $1.55 E-6$ & $3.91 E-7$ & $9.68 E-8$ & $2.36 E-8$ \\
0.375 & $4.72 E-5$ & $1.20 E-5$ & $3.04 E-6$ & $7.71 E-7$ & $1.97 E-7$ \\
0.5 & $2.88 E-4$ & $9.44 E-5$ & $3.45 E-5$ & $1.41 E-5$ & $6.26 E-6$ \\
0.625 & $6.16 E-4$ & $1.60 E-4$ & $4.27 E-5$ & $1.19 E-5$ & $3.65 E-6$ \\
0.750 & $1.70 E-3$ & $4.96 E-4$ & $1.59 E-4$ & $5.76 E-5$ & $2.32 E-5$ \\
0.875 & $3.08 E-3$ & $8.66 E-4$ & $2.64 E-4$ & $9.04 E-5$ & $3.47 E-5$ \\
1 & $2.15 E-3$ & $1.14 E-4$ & $1.84 E-4$ & $1.52 E-4$ & $9.11 E-5$ \\
\hline
\end{tabular}

Table 3: Absolute errors for Example 4.3.

\begin{tabular}{lccccc}
\hline$t_{i}$ & $j=9$ & $j=17$ & $j=33$ & $j=65$ & $j=129$ \\
& $\left|z_{2}\left(t_{i}\right)-x\left(t_{i}\right)\right|$ & $\left|z_{2}\left(t_{i}\right)-x\left(t_{i}\right)\right|$ & $\left|z_{2}\left(t_{i}\right)-x\left(t_{i}\right)\right|$ & $\left|z_{2}\left(t_{i}\right)-x\left(t_{i}\right)\right|$ & $\left|z_{2}\left(t_{i}\right)-x\left(t_{i}\right)\right|$ \\
\hline 0.125 & $3.24 E-4$ & $8.18 E-5$ & $2.08 E-5$ & $5.38 E-6$ & $1.43 E-6$ \\
0.250 & $6.57 E-4$ & $1.73 E-4$ & $4.80 E-5$ & $1.43 E-5$ & $4.74 E-6$ \\
0.375 & $9.34 E-4$ & $2.48 E-4$ & $6.96 E-5$ & $2.12 E-5$ & $7.99 E-6$ \\
0.5 & $2.07 E-3$ & $7.64 E-4$ & $3.13 E-4$ & $1.39 E-4$ & $6.57 E-5$ \\
0.625 & $1.51 E-3$ & $4.60 E-4$ & $1.56 E-4$ & $5.98 E-5$ & $2.53 E-5$ \\
0.750 & $2.22 E-3$ & $7.88 E-4$ & $3.14 E-4$ & $1.37 E-4$ & $6.41 E-5$ \\
0.875 & $2.02 E-3$ & $6.68 E-4$ & $2.65 E-4$ & $1.13 E-4$ & $5.21 E-5$ \\
1 & $6.16 E-3$ & $2.85 E-3$ & $1.37 E-3$ & $6.75 E-4$ & $3.35 E-4$ \\
\hline
\end{tabular}

\section{Acknowledgments}

The research is partially supported by M.E.C. (Spain) and FEDER project no. MTM200612533, and by Junta de Andaluca Grant FQM359. 


\section{References}

[1] Z. Semadeni, "Product Schauder bases and approximation with nodes in spaces of continuous functions," Bulletin de l'Académie Polonaise des Sciences, vol. 11, pp. 387-391, 1963.

[2] B. R. Gelbaum and J. Gil de Lamadrid, "Bases of tensor products of Banach spaces," Pacific Journal of Mathematics, vol. 11, pp. 1281-1286, 1961.

[3] K. Atkinson and W. Han, Theoretical Numerical Analysis: A Functional Analysis Framework, vol. 39 of Texts in Applied Mathematics, Springer, Dordrecht, The Netherlands, 3rd edition, 2009.

[4] C. T. H. Baker, The Numerical Treatment of Integral Equations, Monographs on Numerical Analysis, Clarendon Press, Oxford, UK, 1977.

[5] C. T. H. Baker, "A perspective on the numerical treatment of Volterra equations," Journal of Computational and Applied Mathematics, vol. 125, no. 1-2, pp. 217-249, 2000.

[6] H. Brunner and P. J. van der Houwen, The Numerical Solution of Volterra Equations, vol. 3 of CWI Monographs, North-Holland, Amsterdam, The Netherlands, 1986.

[7] L. M. Delves and J. L. Mohamed, Computational Methods for Integral Equations, Cambridge University Press, Cambridge, Mass, USA, 1985.

[8] Z. Wan, Y. Chen, and Y. Huang, "Legendre spectral Galerkin method for second-kind Volterra integral equations," Frontiers of Mathematics in China, vol. 4, no. 1, pp. 181-193, 2009.

[9] M. I. Berenguer, D. Gámez, A. I. Garralda-Guillem, M. Ruiz Galán, and M. C. Serrano Pérez, "Analytical techniques for a numerical solution of the linear Volterra integral equation of the second kind," Abstract and Applied Analysis, vol. 2009, Article ID 149367, 12 pages, 2009.

[10] G. J. O. Jameson, Topology and Normed Spaces, Chapman and Hall, London, UK, 1974.

[11] P. Hájek, V. Montesinos Santalucía, J. Vanderwerff, and V. Zizler, Biorthogonal Systems in Banach Spaces, CMS Books in Mathematics 26, Springer, New York, NY, USA, 2008.

[12] M. I. Berenguer, M. A. Fortes, A. I. Garralda-Guillem, and M. Ruiz Galán, "Linear Volterra integrodifferential equation and Schauder bases," Applied Mathematics and Computation, vol. 159, no. 2, pp. 495-507, 2004.

[13] D. Gámez, A. I. Garralda-Guillem, and M. Ruiz Galán, "High-order nonlinear initial-value problems countably determined," Journal of Computational and Applied Mathematics, vol. 228, no. 1, pp. 77-82, 2009.

[14] A. Palomares and M. Ruiz Galán, "Isomorphisms, Schauder bases in Banach spaces, and numerical solution of integral and differential equations," Numerical Functional Analysis and Optimization, vol. 26, no. 1, pp. 129-137, 2005. 


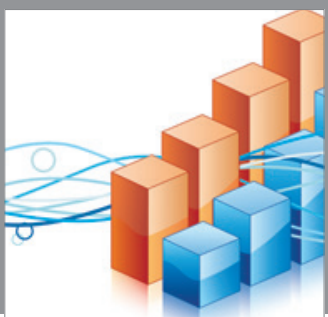

Advances in

Operations Research

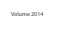

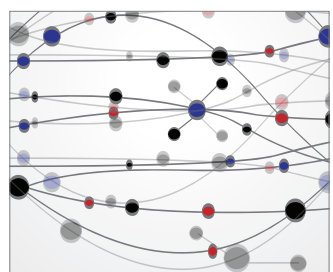

\section{The Scientific} World Journal
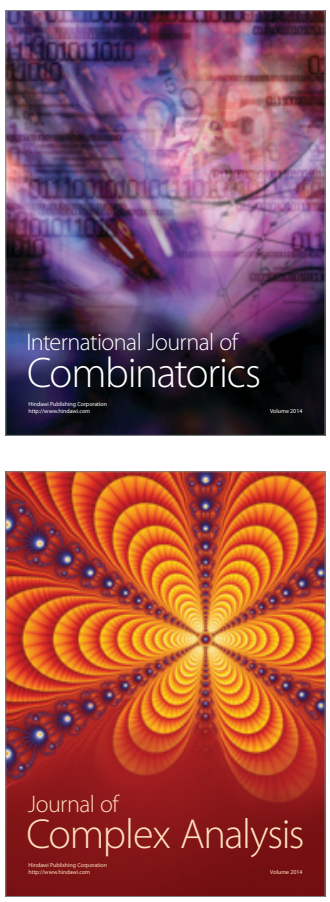

International Journal of

Mathematics and

Mathematical

Sciences
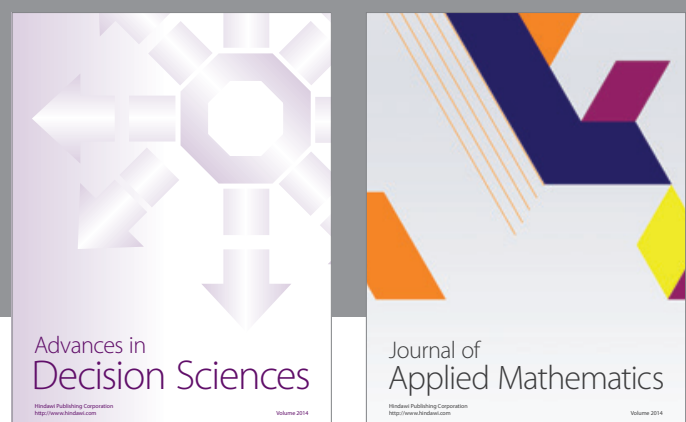

Journal of

Applied Mathematics
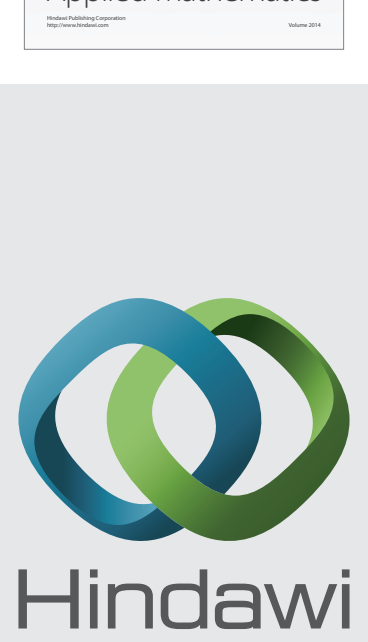

Submit your manuscripts at http://www.hindawi.com
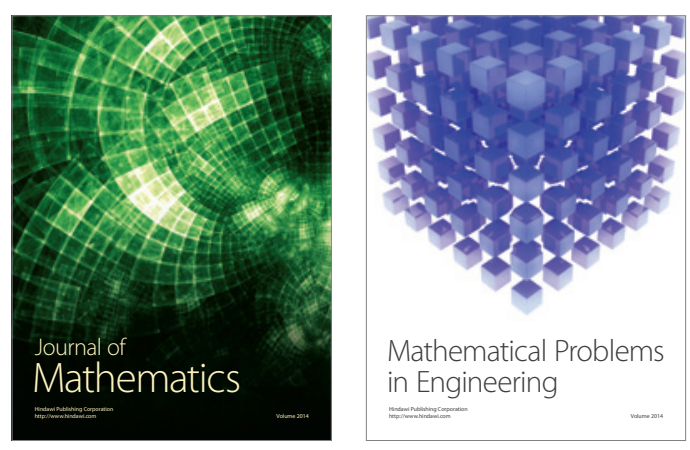

Mathematical Problems in Engineering
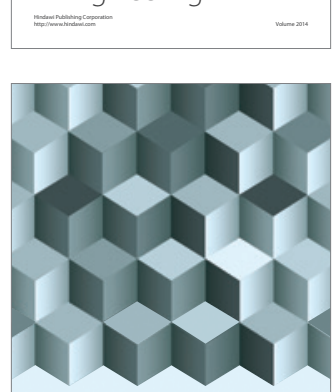

Journal of

Function Spaces
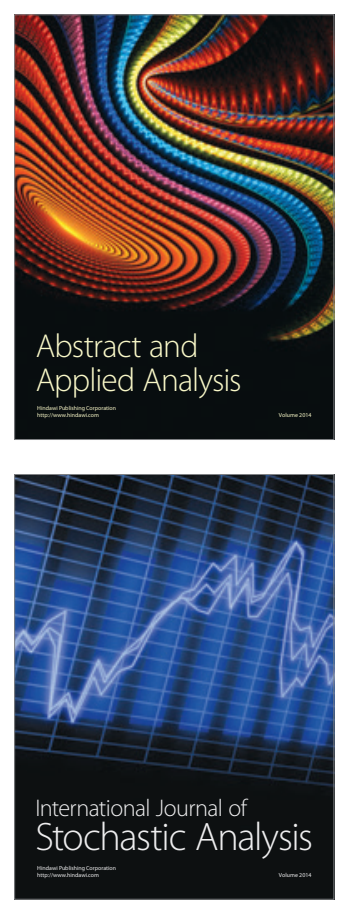

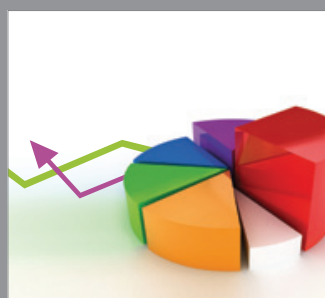

ournal of

Probability and Statistics

Promensencen
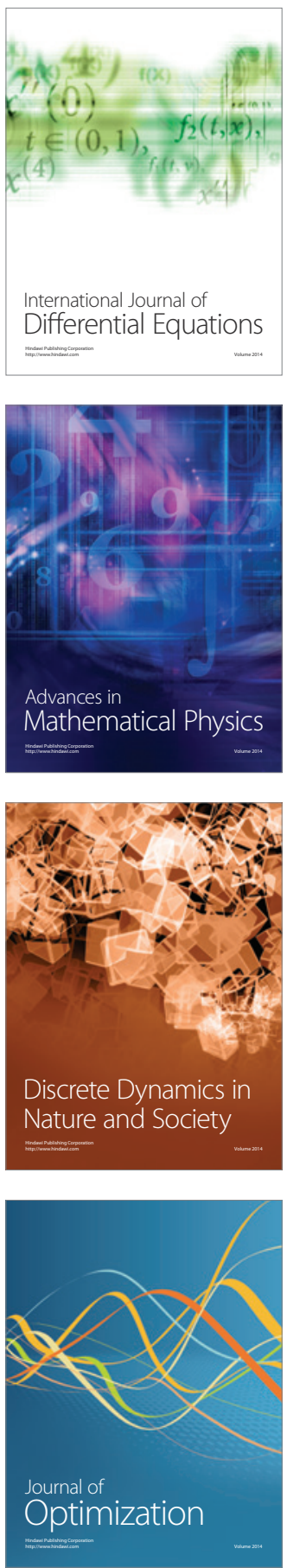